\title{
Star formation rates and the kinematics of gas in the spiral arms of NGC 628
}

\author{
A.S. Gusev ${ }^{1, \star}$, F. Sakhibov ${ }^{2}$, and Yu. N. Efremov ${ }^{1}$ \\ 1 Sternberg Astronomical Institute, Lomonosov Moscow State University, Universitetsky pr. 13, 119992 Moscow, Russia \\ 2 University of Applied Sciences of Mittelhessen, Campus Friedberg, Department of Mathematics, Natural Sciences and \\ Data Processing, Wilhelm-Leuschner-Strasse 13, 61169 Friedberg, Germany
}

Received 2015 Jan 20, accepted 2015 Apr 13

Published online XXXX

Key words galaxies: individual (NGC 628) - HII regions - ISM: kinematics and dynamics

Relations between star formation rates along the spiral arms and the velocities of gas inflow into the arms in grand-design galaxy NGC 628 were studied. We found that the radial distribution of average star formation rate in individual star formation regions in regular spiral arms correlates with the velocity of gas inflow into the spiral arms. Both distributions have maxima at a galactocentric distance of $4.5-5 \mathrm{kpc}$. There are no correlations between the radial distributions of average star formation rate in star formation regions in spiral arms and outside spiral arms in the main disc. We also did not find a correlation between the radial distribution of average star formation rate in star formation regions in spiral arms and $\mathrm{H} \mathrm{I}$ column density.

Copyright line will be provided by the publisher

\section{Introduction}

A connection between star formation and the spiral structure of disc galaxies is known since Morgan, Whitford \& Code (1953) found concentrations of OB stars in the Sagittarius spiral arm of the Milky Way. This connection suggests that spiral arms trigger star formation. The development of the density-wave theory has shed new light on the connection between star formation and spiral arms. A density wave traveling through the disc of a galaxy produces a shock in the gas, which should trigger formation of stars in spiral arms. Spiral arms are dense and promote more gravitational instabilities and cloud collisions than the interarm regions, triggering molecular cloud formation and conglomeration in the arms. This can easily explain the observed concentration of young stars in spiral arms.

Many studies of the spiral structure in galaxies compare various observational data in order to link star formation with spiral density waves in discs. These comparisons are mostly qualitative. There is very illustrative diagram of Roberts, Roberts \& Shu (1975), which relates the expected velocity of gas inflow into the spiral arm and the magnitude of the spiral arm shock with the morphological characteristics of the spiral structure and van den Bergh luminosity classes. The earlier the luminosity class of the galaxy (i.e., the higher its luminosity), the brighter and more conspicuous the spiral pattern it possesses. In this case, the galactic gas meets the spiral arm at a higher velocity. The higher this velocity, the stronger the shock of gas and the higher the luminosity of the galaxy.

^ Corresponding author: e-mail: gusev@ sai.msu.ru
A more detailed analysis one can make through the study of non-circular motions of gas clouds and stars caused by the spiral density wave in the disc of a galaxy. The perturbed velocities due to the spiral wave depend on the amplitude of the wave, the position of the corotation and Lindblad resonances, and the mass distribution in the galaxy (the shape of rotation curve). The same parameters affect the radial behaviour of the velocity of gas inflow into the spiral arm, and thereby probably also the radial profile of SFR in the disc.

Previously we compared a variation of the velocity of gas inflow, $v_{\perp}$, into the spirals and the surface density of star formation rate, $\Sigma_{\mathrm{SFR}}$, in the disc with the galactocentric distance $r$ in the spiral galaxy NGC 628 (Sakhibov \& Smirnov 2004). We believe that the radial distribution of the SFR surface density, $\Sigma_{\mathrm{SFR}}$, over the disc has a ring form with the maximum at the galactocentric distance $r \sim 3 \mathrm{kpc}$, whereas the maximum of the velocity of gas inflow $v_{\perp}$ is situated at $r \sim 5 \mathrm{kpc}$. We derived the velocities of gas inflow $v_{\perp}$ into the spirals at different galactocentric distances via a Fourier analysis of the azimuthal distribution of the observed radial velocities in annular (ring form) zones of the disc of the NGC 628 and the radial profile of the surface density of SFR, using the empirical 'SFR vs. linear size' relation for star formation complexes (Sakhibov \& Smirnov 2004) and coordinates, $\mathrm{H} \alpha$ fluxes, and the sizes of $\mathrm{H}$ II regions presented by Belley \& Roy (1992).

The deviation between the maximum of the $\Sigma_{\mathrm{SFR}}$ in the disc at the $r \sim 3 \mathrm{kpc}$ and the maximum of the velocity of gas inflow $v_{\perp}$ at $r \sim 5 \mathrm{kpc}$ shows that the total galactic star formation rate in the main disc is not significantly enhanced by the presence of spiral arms (see Elmegreen 2010). 


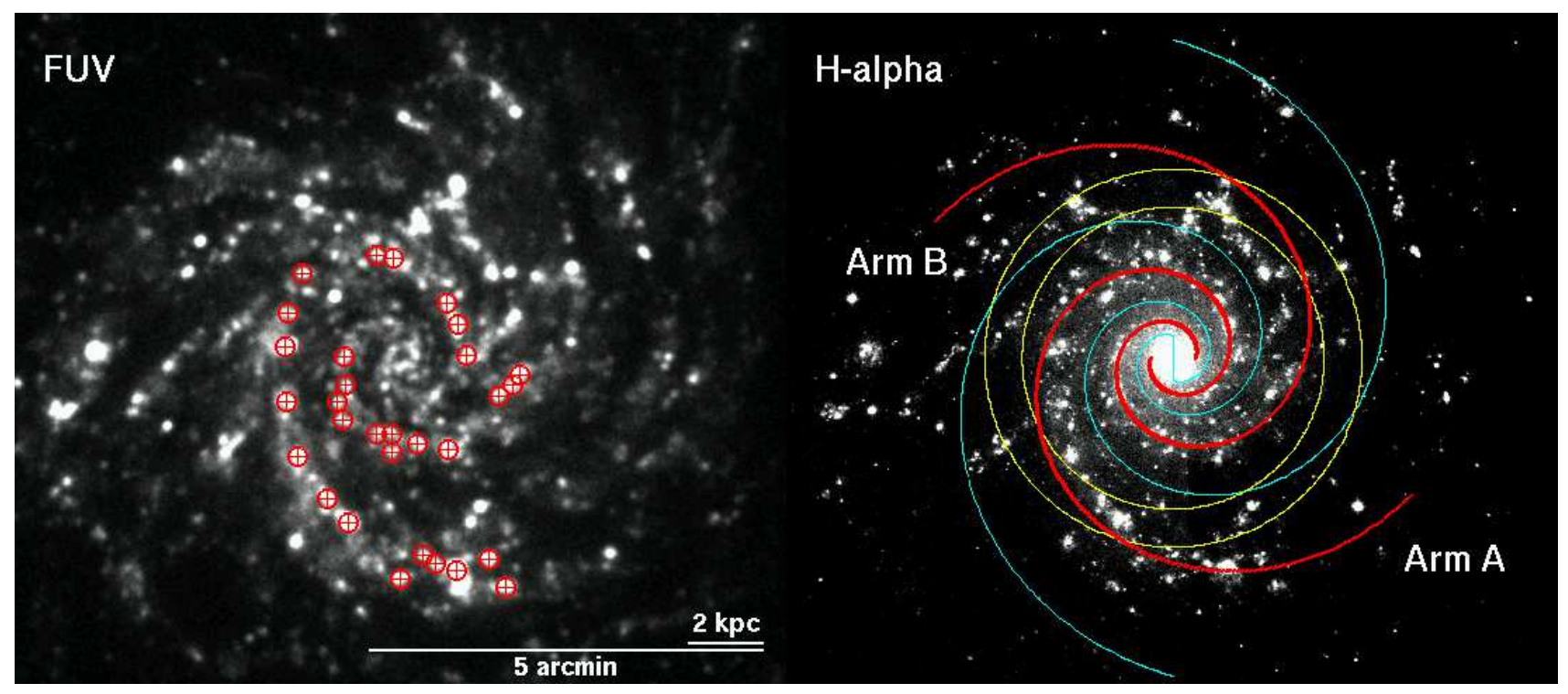

Fig. 1 (online colour at: www.an-journal.org) Images of NGC 628 in the FUV (left) and H $\alpha$ line (right). The FUV image was taken from GALEX archive, the $\mathrm{H} \alpha$ image was obtained on the Maidanak Observatory with the $1.5 \mathrm{~m}$ telescope. Positions of star formation regions (red crosses in circles) from the list of Gusev et al. (2014) are indicated in the FUV image. Logarithmic spiral arms (thick red curves) and the boundary between two parts of the galaxy, around Arm A and Arm B (thin cyan curve) are shown in the $\mathrm{H} \alpha$ line image. Yellow circles in the $\mathrm{H} \alpha$ image have radii $r=4$ and $5 \mathrm{kpc}$ (see the text for details). North is upward and east is to the left.

Table 1 Basic parameters of NGC 628.

\begin{tabular}{ll}
\hline Parameter & Value \\
\hline Type & $\mathrm{SA}(\mathrm{s}) \mathrm{c}$ \\
Total apparent $B$ magnitude $\left(B_{t}\right)$ & $9.70 \pm 0.26 \mathrm{mag}$ \\
Absolute $B$ magnitude $\left(M_{B}\right)^{a}$ & $-20.72 \mathrm{mag}$ \\
Inclination $(i)$ & $7^{\circ} \pm 1^{\circ}$ \\
Position angle (PA) & $25^{\circ}$ \\
Heliocentric radial velocity $(v)$ & $659 \pm 1 \mathrm{~km} \mathrm{~s}^{-1}$ \\
Apparent corrected radius $\left(R_{25}\right)^{b}$ & $5.23 \pm 0.24 \mathrm{arcmin}$ \\
Apparent corrected radius $\left(R_{25}\right)^{b}$ & $10.96 \pm 0.51 \mathrm{kpc}$ \\
Distance $(d)$ & $7.2 \mathrm{Mpc}$ \\
Galactic absorption $\left(A(B)_{\mathrm{Gal}}\right)$ & $0.254 \mathrm{mag}$ \\
Distance modulus $(m-M)$ & $29.29 \mathrm{mag}$ \\
\hline
\end{tabular}

${ }^{a}$ Absolute magnitude of the galaxy corrected for Galactic extinction and inclination effect.

${ }^{b}$ Isophotal radius (25 mag $\operatorname{arcsec}^{-2}$ in the $B$-band) corrected for Galactic extinction and absorption due to the inclination of NGC 628 .

But the question, whether the peak of the velocity of gas inflow $v_{\perp}$ into the spirals coincides with the peak of the radial distribution of the SFR within the spirals in the individual star formation regions, remains open.

Recently Gusev \& Efremov (2013) and Gusev, Egorov \& Sakhibov (2014) investigated photometric properties of spiral arms in NGC 628, and location and parameters of star formation regions inside these arms. Thirty brightest star formation regions in the ultraviolet band, located in the spiral arms of NGC 628, were identified and studied (see Fig. 11). We found that the star formation regions in Arm A are systematically brighter and larger than the re- gions in Arm B (Gusev et al. 2014). We also measured the star formation rates (SFRs) and the surface densities of SFR $\left(\Sigma_{\mathrm{SFR}}\right)$ within these star formation regions using obtained FUV magnitudes, $\mathrm{H} \alpha$ luminosities and sizes. Since the star formation rate per unit area is higher in the arms than in the main disc we decide to study relations between SFRs along the arms and the velocities of gas inflow into the spiral arms.

The main goal of this new research is to study relations between SFRs along the arms and the velocities of gas inflow into the spiral arms. This study is based on our own observations of the galaxy in $\mathrm{H} \alpha$ line, obtained on the Maidanak Observatory with the $1.5 \mathrm{~m}$ telescope, Galaxy Evolution Explorer (GALEX) far-ultraviolet (FUV) data (Gusev \& Efremov 2013; Gusev et al. 2014), as well as a Fourier analysis of the spatial distribution of the radial velocities of the neutral gas in the disc (Sakhibov \& Smirnov 2004).

Observations, data reduction, determination of parameters of spiral arms, selection criteria and photometry of star formation regions have been described in detail in Gusev \& Efremov (2013) and Gusev et al. (2014).

\section{NGC 628, previous studies}

The spiral galaxy NGC 628 (M74) is one of the best studied nearby spiral galaxies viewed almost face-on (Fig. 1 , Table 11. This prototypical grand-design galaxy hosts two principal arms. The first (South arm by Rosales-Ortega et al. 2011) is a long regular arm (Arm A in Fig. 11). In this arm Elmegreen \& Elmegreen (1983) found a regular string 
of star complexes (H II regions). The shorter second arm (Arm B in Fig. 11) have the distorted outer part.

NGC 628 is an excellent example of a galaxy that has experienced recent star formation episodes. Hodge (1976) identified $730 \mathrm{H}$ II regions in the galaxy. Ivanov et al. (1992) observed and compiled a catalogue of positions, angular sizes, integral magnitudes and $\mathrm{H}$ II identification of $147 \mathrm{OB}$ associations and aggregates in NGC 628. Numerous star formation regions have been studied earlier based on photometric, spectroscopic and spectrophotometric observations (McCall, Rybski \& Shields 1985; Belley \& Roy 1992; Ferguson, Gallagher \& Wyse 1998; Bresolin, Kennicutt \& Garnett 1999; Larsen 1999, 2004; Bruevich et al. 2007; RosalesOrtega et al. 2011; Gusev et al. 2012; Berg et al. 2013).

Kinematics of the H I disc has been studied by Shostak \& van der Kruit (1984) and Kamphuis \& Briggs (1992). The neutral hydrogen distribution correlates with the optical structure within the optical disc. The H I velocity field out of the optical edge can be ascribed to circular rotation in a plane (Shostak \& van der Kruit 1984). Two giant high velocity gas complexes $\left(M(\mathrm{HI}) \sim(0.5-1) \times 10^{8} M_{\odot}\right)$ are located at $\sim 10$ arcmin to the east and to the west from the galactic centre (Kamphuis \& Briggs 1992). The gas velocity dispersion, as well as the stellar dispersion are typical for giant spiral galaxies, $\sigma(\mathrm{H} \mathrm{I})=8-10 \mathrm{~km} \mathrm{~s}^{-1}$ at galactocentric distances, $r$, of $1.5-3.5 \operatorname{arcmin}(3.0-7.5 \mathrm{kpc}$ for an adopted distance to NGC 628 of $7.2 \mathrm{Mpc}$; Shostak $\&$ van der Kruit 1984), $\sigma(\mathrm{H} \mathrm{II})=16-19 \mathrm{~km} \mathrm{~s}^{-1}$ at $r$ of $0.5-2.7 \operatorname{arcmin}(1.0-5.5 \mathrm{kpc})$ and $14 \pm 7 \mathrm{~km} \mathrm{~s}^{-1}$ at $r$ of $2.8-4.0$ arcmin (6.0-8.5 kpc; Fathi et al. 2007), and $\sigma($ stars $)=50 \pm 20 \mathrm{~km} \mathrm{~s}^{-1}$ at $r$ of $0.3-1.0 \operatorname{arcmin}(0.7-$ 2.0 kpc; MacArthur, González \& Courteau 2009).

Although NGC 628 is a member of a small group of galaxies (Auld et al. 2006), it cannot have undergone any encounter with satellites or other galaxies in the past $1 \mathrm{Gyr}$ (Wakker \& van Woerden 1991; Kamphuis \& Briggs 1992).

The rotation speed of the arms (pattern speed) is determined along with other parameters of the spiral-density wave (Sakhibov \& Smirnov 2004) via a Fourier analysis of the azimuthal distribution of the observed radial velocities (Shostak \& van der Kruit 1984) in annular zones of the disc of NGC 628. Comparison of the pattern speed with the pure circular motion of the gas provided the velocity of gas inflow into the spiral arm at different radial distances. Corresponding corotation radius locates at $R_{\text {cor }} \approx 7 \mathrm{kpc}$ (Sakhibov \& Smirnov 2004).

The fundamental parameters of NGC 628 are presented in Table 1. We take the distance to NGC 628, obtained in Sharina, Karachentsev \& Tikhonov (1996) and van Dyk, Li \& Filippenko (2006). We used the position angle and the inclination of the galactic disc, derived by Sakhibov \& Smirnov (2004). The morphological type and the Galactic absorption, $A(B)_{\text {Gal }}$, are taken from the NED data base 11 Other parameters are taken from the LEDA data base 2 (Paturel et

\footnotetext{
1 http://ned.ipac.caltech.edu/

2 http://leda.univ-lyon1.fr/
}

al. 2003). We adopt the Hubble constant $H_{0}=75 \mathrm{~km} \mathrm{~s}^{-1} \mathrm{Mpc}^{-1}$. With the assumed distance to NGC 628, we estimate a linear scale of $34.9 \mathrm{pc} \mathrm{arcsec}^{-1}$.

\section{Radial distributions of the star formation rates}

We derived the radial distributions of SFR in the galaxy using FUV and $\mathrm{H} \alpha$ images of NGC 628. Previously, all data were corrected for Galactic absorption. Here we used the resulting ratio of the extinction in the GALEX FUV band to the color excess $E(B-V) A_{\mathrm{FUV}} / E(B-V)=8.24$ (Wyder et al. 2007) and the standard ratio $c(\mathrm{H} \alpha)=0.308 A(V)$.

Azimuthally averaged photometric profiles of the galaxy in FUV and $\mathrm{H} \alpha$ were obtained using round apertures in steps of 2 arcsec. We do not transform images of NGC 628 to the face-on position, $i=0^{\circ}$, because the position of any point in the galaxy is changed by value much less than the characteristic width of the spiral arm, if we use the adopted inclination $i=7^{\circ}$. Difference between the uncorrected position and the position corrected for the inclination position angle is less than $0.5^{\circ}$ and the galactocentric distance $\Delta r / r<0.01$ for any point in the galaxy.

For estimation of SFR radial distribution peculiarities in the two opposite spiral arms of NGC 628, we divided the galactic image into two equal parts. The first part includes the area around Arm A, and the second part - around Arm B. We built the boundary between the two parts using the parameters of symmetric logarithmic spirals obtained in Gusev \& Efremov (2013). We determined the equation of the boundary at galactocentric distances $r \geq 21.95 \operatorname{arcsec}$ as the equations of logarithmic spiral Arms A and B (see Gusev \& Efremov 2013) that are rotated by $90^{\circ}$ in the galactic plane. For the inner part of NGC 628, at $r<21.95$ arcsec, the boundary lies on the north-south line (Fig. 11).

We adopt the conversion factor of FUV luminosity to the star formation rate of Iglesias-Páramo et al. (2006).

$$
\operatorname{SFR}\left(M_{\odot} \mathrm{yr}^{-1}\right)=8.13 \times 10^{-44} L_{\mathrm{FUV}}\left(\mathrm{erg} \mathrm{s}^{-1}\right),
$$

and the conversion factor of $\mathrm{H} \alpha$ luminosity to star formation rate of Kennicutt (1998)

$$
\operatorname{SFR}\left(M_{\odot} \mathrm{yr}^{-1}\right)=7.9 \times 10^{-42} L_{\mathrm{H} \alpha}\left(\mathrm{erg} \mathrm{s}^{-1}\right) .
$$

To estimate physical values of SFR along the spiral arms, we introduce the term 'SFR per unit of length of a spiral arm', $\Delta \mathrm{SFR} / \Delta l$. We adopt here, that all FUV and $\mathrm{H} \alpha$ emission is concentrated in the thin spiral arms of the galaxy.

For a logarithmic spiral with a pitch angle $\mu$, the longitudinal displacement segment along the spiral, $\Delta l$, within a ringed aperture with a width $\Delta r$ is

$$
\Delta l=\Delta r / \sin \mu
$$

independently of the galactocentric radius. Gusev \& Efremov (2013) obtained a pitch angle $\mu=15.7^{\circ}$ for the arms. It is consistent with the estimations by Danver (1942) $\mu=$ $17^{\circ} \pm 2^{\circ}$ and close to the result of Kennicutt \& Hodge (1976) 

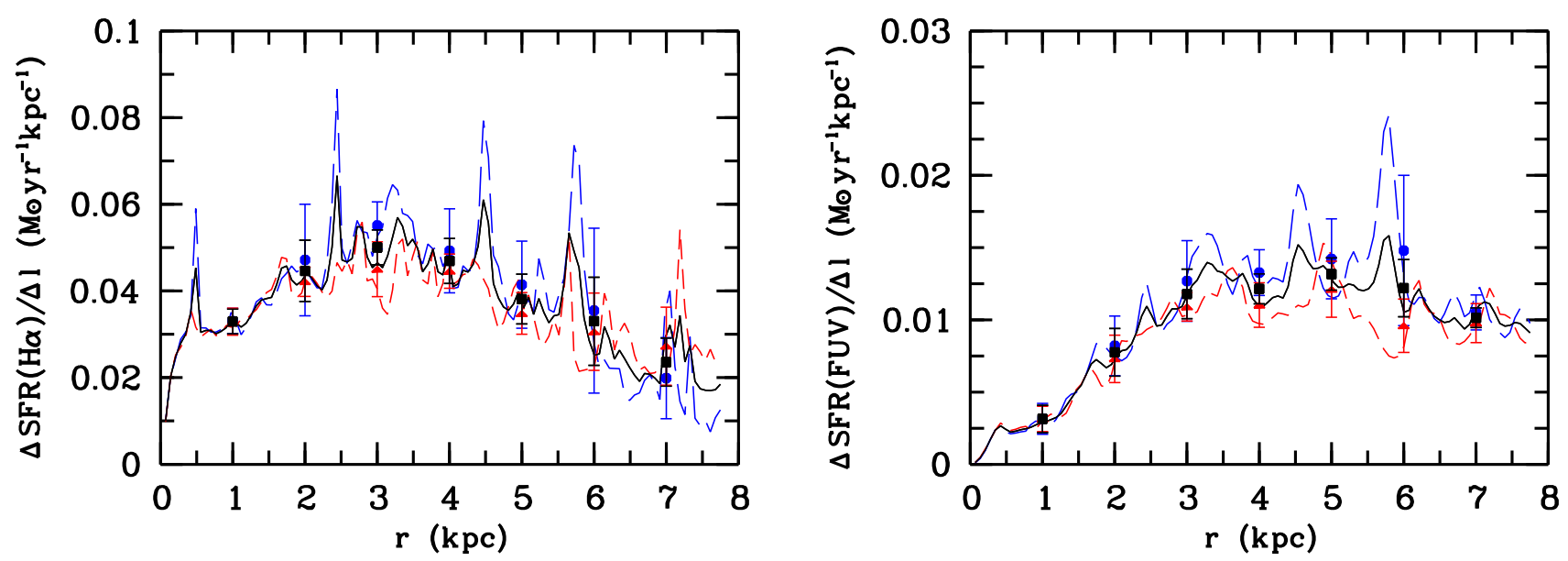

Fig. 2 (online colour at: www.an-journal.org) Radial distributions of SFRs per the unit of length of spiral arm, $\Delta \mathrm{SFR} / \Delta l$, based on the luminosities in $\mathrm{H} \alpha$ (left panel) and FUV (right panel), and their averaged values for Arm A (blue long-dashed curves and circles), Arm B (red short-dashed curves and triangles), and the mean for both arms (black solid curves and squares). The $\Delta \mathrm{SFR} / \Delta l$ averaged error bars are shown.

who fitted every arm separately and obtained values $13.8^{\circ}$ for Arm A and $11.2^{\circ}$ for Arm B.

Thus, the values of $\Delta \mathrm{SFR} / \Delta l$ were obtained using Eqs. (1), (2) and (3), and the constants $r=2 \operatorname{arcsec}(70 \mathrm{pc})$ and $\mu=15.7^{\circ}$.

The radial distributions of $\Delta \mathrm{SFR} / \Delta l$ are shown in Fig.2 for Arms A and B separately. We also give the mean radial distributions for both arms in this figure. Additionally, we calculated the values of $\Delta \mathrm{SFR} / \Delta l$, averaged over $r$ in steps of $1 \mathrm{kpc}$. These data are also presented in Fig. 3

The radial distributions show slightly higher SFR in Arm A than in Arm B at galactocentric distances from 2 to 6 kpc. However, the distributions based on FUV luminosities differ significantly from ones based on $\mathrm{H} \alpha$ emissions. The distribution of $\Delta \mathrm{SFR}(\mathrm{H} \alpha) / \Delta l$ has clear maximum at $r \approx 3-4 \mathrm{kpc}$, the distribution of $\Delta \mathrm{SFR}(\mathrm{FUV}) / \Delta l$ has a flat plot at $r=3-6 \mathrm{kpc}$ (Fig. 3).

The values of $\Delta \operatorname{SFR}(\mathrm{H} \alpha) / \Delta l$ are 2-10 times larger than the values of $\Delta \mathrm{SFR}(\mathrm{FUV}) / \Delta l$, this ratio decreases towards the outer part of NGC 628 (Fig. 3). These differences may be explained by the radial gradient of dust absorption in the disc of the galaxy. We roughly estimated parameters of dust distribution based on the condition

$$
\operatorname{SFR}(\mathrm{FUV}) / \operatorname{SFR}(\mathrm{H} \alpha)(r)=1 \text {. }
$$

The difference between FUV and $\mathrm{H} \alpha$ luminosity distributions shown in Fig. 3 can be explained by the gradient of dust absorption

$$
A(B)=1.545-0.134 r,
$$

where $r$ is the galactocentric distance in units of kpc. If the dust is uniformly mixed in the stellar and gaseous medium, the dust absorption, $A(B)$, depends on the dust opacity, $\tau_{B}$, as

$$
A(B)=-2.5 \log \left[1-\exp \left(-\tau_{B}\right)\right] / \tau_{B}
$$

(Disney, Davies \& Phillipps 1989). Using Eqs. (4) and (5) we estimated the central opacity in the $B$ band, $\tau_{B} \approx 3.9$, and the scale length of the dust disc, $h(B)_{\text {dust }} \approx 3.2 \mathrm{kpc}$. The value of $h(B)_{\text {dust }}$ is close to the disc scale length in the $B$ passband obtained by Möllenhoff (2004), who estimated $h(B)=3.11 \mathrm{kpc}$. The value of the central opacity in $B$ is typical for dust opacities in central regions of local universe spiral galaxies according to Popescu et al. (2011). who found $\tau_{B} \sim 3.5-4$. We also estimated the total dust mass using Eq. (44) from Popescu et al. (2011), $M_{\text {dust }} \approx$ $4 \times 10^{7} M_{\odot}$. It is close to the last estimation of the dust mass in NGC 628. Aniano et al. (2012) found that $M_{\text {dust }}=$ $(2.9 \pm 0.4) \times 10^{7} M_{\odot}$ using the data of Spitzer and Hershel (from $3.6 \mu \mathrm{m}$ to $500 \mu \mathrm{m}$ ).

Nevertheless note that the radial distribution of the velocities of gas inflow into the spiral arms, $v_{\perp}$, poorly correlates with the distributions of SFRs based on both FUV and $\mathrm{H} \alpha$ luminosities (Fig. 3).

\section{Star formation rates within star formation regions and the radial distribution of the velocity of gas inflow into the spiral arms}

To obtain the SFR, free from the dust influence, we used the data of SFR within star formation regions, which were obtained for 30 largest young stellar objects with known interstellar absorption in the regular part of spiral arms of NGC 628 in Gusev et al. (2014). Gusev et al. (2014) selected 30 star formation regions having a total magnitude corrected for interstellar absorption $\mathrm{FUV}_{0}<19.8 \mathrm{mag}$. Among regions fainter than 19.8 mag in the FUV, we found a large number of diffuse objects without strong $\mathrm{H} \alpha$ emission. Moreover, there are no measurements of interstellar absorption for such faint objects. We divided the star formation re- 


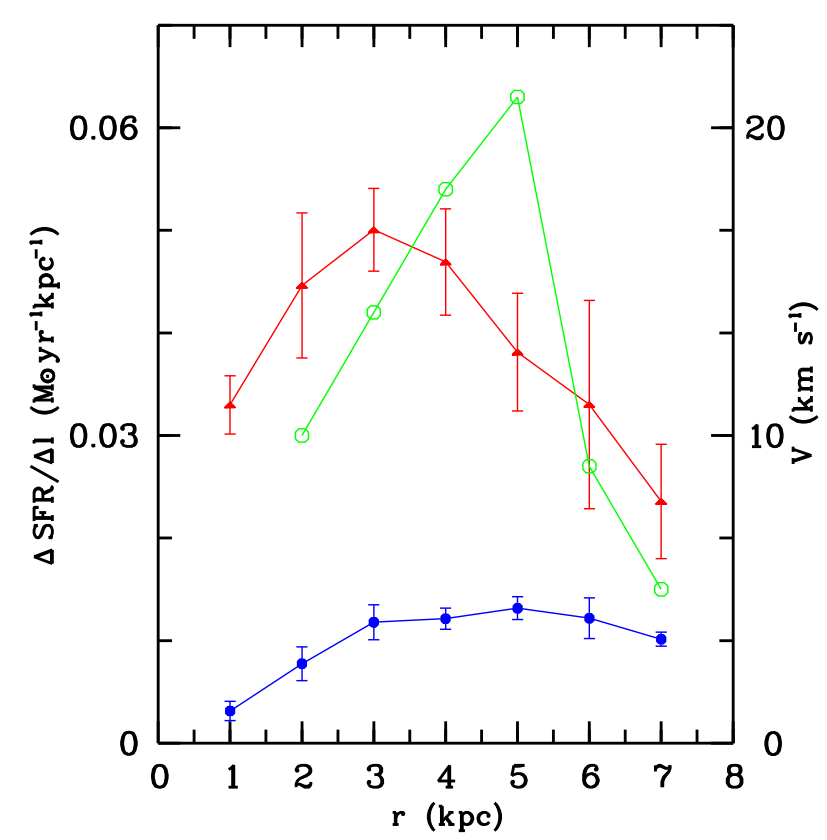

Fig. 3 (online colour at: www.an-journal.org) Radial distributions of averaged values of $\Delta \mathrm{SFR} / \Delta l$ based on the luminosities in $\mathrm{H} \alpha$ (red line and triangles) and FUV (blue line and circles) for both arms. The $\Delta \mathrm{SFR} / \Delta l$ averaged error bars are shown. Radial distributions of the velocities of gas inflow into the spiral arms (green line and open circles) by Sakhibov \& Smirnov (2004) are shown.

gions into several groups depending on their distances to the galactic centre with a step of $1 \mathrm{kpc}$. For every group, the average SFR, $\langle\mathrm{SFR}\rangle$, was calculated as

$$
\langle\mathrm{SFR}\rangle=\frac{1}{n} \sum_{n} \mathrm{SFR}_{n},
$$

where $n$ is a number of objects in the group and $\mathrm{SFR}_{n}$ is the SFR within the $n^{t h}$ object. These mean SFRs were obtained separately for the objects in Arm A and Arm B based on both FUV and $\mathrm{H} \alpha$ data (Fig. 4). We also calculated the mean SFRs for both FUV and $\mathrm{H} \alpha$ luminosities as $(\langle\mathrm{SFR}(\mathrm{FUV})\rangle+\langle\operatorname{SFR}(\mathrm{H} \alpha)\rangle) / 2$.

Note that the total SFR within studied 30 regions, $\approx$ $0.25 M_{\odot} \mathrm{yr}^{-1}$, is a significant part (one third) of the total SFR in NGC 628, $0.7 \pm 0.2 M_{\odot} \mathrm{yr}^{-1}$ (Calzetti et al. 2010).

Similarly we calculated the mean values of SFR surface density within the star formation regions, $\left\langle\Sigma_{\mathrm{SFR}}\right\rangle$. They are measured as

$$
\left\langle\Sigma_{\mathrm{SFR}}\right\rangle=\frac{4}{\pi}\left\langle\frac{\mathrm{SFR}}{d^{2}}\right\rangle
$$

where diameters of star formation regions, $d$, were obtained in Gusev et al. (2014).

Arm B is distorted at the galactocentric distances $r>$ $3.2 \mathrm{kpc}$ (Fig. 1). We did not measure the luminosities of star formation regions in the distorted part of Arm B. As a result, the radial distributions of $\langle\mathrm{SFR}\rangle$ and $\left\langle\Sigma_{\mathrm{SFR}}\right\rangle$ within
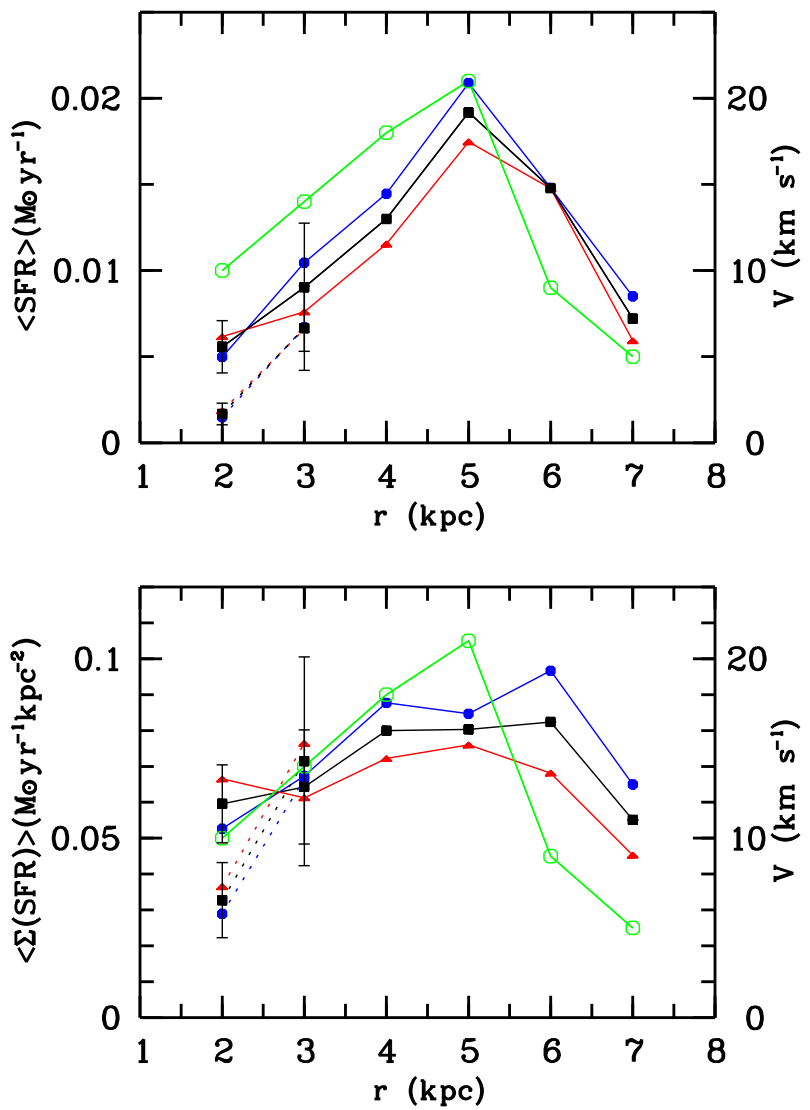

Fig. 4 (online colour at: www.an-journal.org) Radial distributions of the mean SFR (top) and the mean surface density of SFR (bottom) within star formation regions based on their luminosities in FUV (blue lines and circles), $\mathrm{H} \alpha$ (red lines and triangles), and the mean for both luminosities (black lines and squares) in Arm A (solid lines) and Arm B (dotted lines). Radial distributions of the velocities of gas inflow into the spiral arms (green solid curves and open circles) are shown. The $\langle\mathrm{SFR}\rangle$ and $\left\langle\Sigma_{\mathrm{SFR}}\right\rangle$ error bars are shown for Arms A and B.

the star formation regions in Arm B were constructed up to $r \approx 3 \mathrm{kpc}$ only.

As seen from Fig. 4 the values and the distributions of $\langle\mathrm{SFR}\rangle$ and $\left\langle\Sigma_{\mathrm{SFR}}\right\rangle$ obtained from $\mathrm{FUV}$ and $\mathrm{H} \alpha$ data are close to each other. The mean SFR within star formation regions in Arm A is comparable within the errors with the mean SFR within star formation regions in Arm B.

Distribution of the mean SFR within star formation regions in Arm A correlates with the distribution of the velocities of gas inflow into the spiral arm very well. Both distributions have clear maximum at $r=5 \mathrm{kpc}$. The mean surface density of SFR within star formation regions does not correlate with $v_{\perp}$, it is approximately constant at $r=$ $3-6 \mathrm{kpc},\left\langle\Sigma_{\mathrm{SFR}}\right\rangle=0.06-0.09 \mathrm{Myr}^{-1} \mathrm{kpc}^{-2}$, and it falls down towards corotation region of NGC 628 (Fig. (4). 

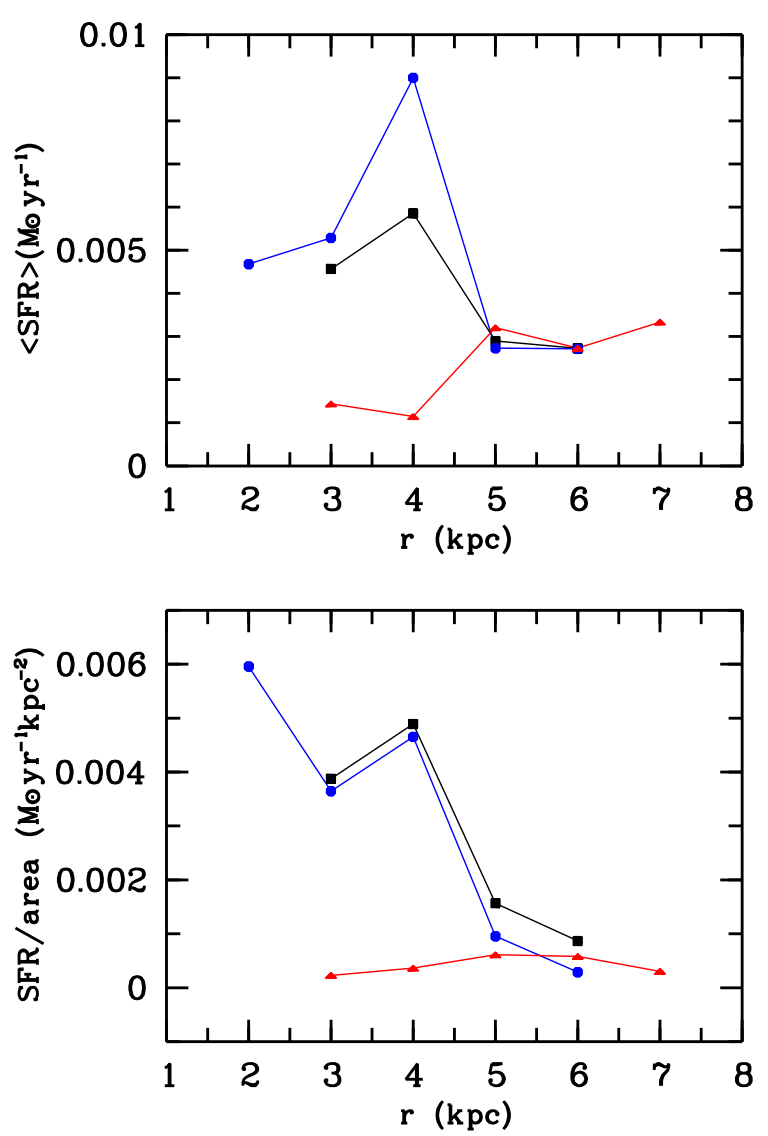

Fig. 5 (online colour at: www.an-journal.org) Radial distributions of the mean SFR (top) and the total SFR per square $\mathrm{kpc}$ (bottom) within star formation regions based on their luminosities in $\mathrm{H} \alpha$ by Rosales-Ortega et al. (2011). Distributions of SFR within star formation regions in spiral arms (blue lines and circles), within star formation regions outside spiral arms in the main disc (red lines and triangles), and within all star formation regions (black lines and squares) are shown.

Difference between the radial distributions of $\langle\mathrm{SFR}\rangle$ and $\left\langle\Sigma_{\mathrm{SFR}}\right\rangle$ is a result of the fact that the star formation regions in Arm A on the galactocentric distances $4 \mathrm{kpc}<r<6 \mathrm{kpc}$ are larger and brighter than those in the inner and outer parts of the arm.

A small number of objects in our sample, containing only bright star formation regions, makes it impossible to determine the maximum in the radial distribution of SFR with an accuracy less than $\pm 1 \mathrm{kpc}$. That is why we additionally examined a sample of $\mathrm{H}$ II regions from Rosales-Ortega et al. (2011) containing 96 objects inside and outside the spiral arms of the galaxy. The results of the analysis of this sample are presented in Fig. 5. Here, we calculated the average SFR using Eq. (6). In contrast to the bottom panel of Fig. 4 where we plotted the profile of the mean SFR within star formation regions per area occupied by a star formation region, calculated by Eq. (7), in the bottom panel of Fig. 5 we present the profile of the total SFR within star formation regions per the galaxy area, averaged by $r$,

$$
\mathrm{SFR} / \text { area }=\sum_{n} \mathrm{SFR}_{n} / \pi\left[(r+0.5)^{2}-(r-0.5)^{2}\right],
$$

where $r$ is a galactocentric distance in units of $\mathrm{kpc}$.

Only two objects in the spiral arms have $r=7 \pm 0.5 \mathrm{kpc}$ and two star formation regions outside the spiral arms are located at distance $r=2 \pm 0.5 \mathrm{kpc}$. We do not show data for them in Fig. 5 .

As seen from Figs. 4 and 5 the maximum in distribution of the mean SFR within star formation regions in spiral arms by Rosales-Ortega et al. (2011) is shifted relative to the maximum in distribution of the mean SFR within star formation regions in Arm A by our data (4 kpc versus $5 \mathrm{kpc}$ ). The basic reason of this shift is a presence of several bright H II complexes, which are inside Arm B in the northern-western part of NGC 628; these complexes are located at $r \approx 4-4.5 \mathrm{kpc}$ (Fig. 1). The largest and brightest in H $\alpha$ complex No. 40 by Rosales-Ortega et al. (2011) is also located here. Moreover, a significant contribution to the increase in $\langle\mathrm{SFR}\rangle$ is made by one of the brightest star formation regions, No. A12 (Gusev et al. 2014) $=84$ (RosalesOrtega et al. 2011), which is located at $r \approx 4.5 \mathrm{kpc}$. The estimated galactocentric distance to his centre is $4.60 \mathrm{kpc}$, however Rosales-Ortega et al. (2011) gave the distance $r=$ $4.43 \mathrm{kpc}$.

The shift in absolute values of the mean SFR between the distributions based on our data and the data of RosalesOrtega et al. (2011) is due to the fact that the sample of Rosales-Ortega et al. (2011) contains more H II regions, than ours, and includes fainter regions not included in our sample.

Note that the maximal velocity of gas inflow into the spiral arms is apparently achieved at distances $r \sim 4.5-$ $5.0 \mathrm{kpc}$, as can be seen from the velocity graph in Fig. 4

Total SFR within star formation regions in spiral arms per the galaxy area decreases considerably with increasing distance from the centre of the galaxy (bottom panel of Fig. 5). Nevertheless, the increase in star formation rate at $r=4 \mathrm{kpc}$ seen clearly in the figure.

Radial distributions of the mean and total SFRs within star formation regions inside and outside the spiral arms are significantly different each from other (Fig. 5). Both $\langle\mathrm{SFR}\rangle$ and total SFR per galaxy area within star formation regions in spiral arms have obvious maxima at $r \approx 4 \mathrm{kpc}$ and they decrease towards $r \sim 6 \mathrm{kpc}$. 〈SFR〉 and the total SFR within star formation regions outside the spiral arms grow on the distances from 4 to $6 \mathrm{kpc}$ (Fig. 5). Both $\langle\mathrm{SFR}\rangle$ and total SFR per galaxy area within star formation regions become equal to each other for the objects inside and outside the spiral arms at $r \approx 6 \mathrm{kpc}$.

Obviously, star formation parameters depend on the properties of interstellar medium. The key ingredient of star formation is a molecular hydrogen. However, molecular hydrogen is concentrated in the central part of galaxies. In contrast to $\mathrm{H}_{2}$, a density of neutral hydrogen decreases towards 


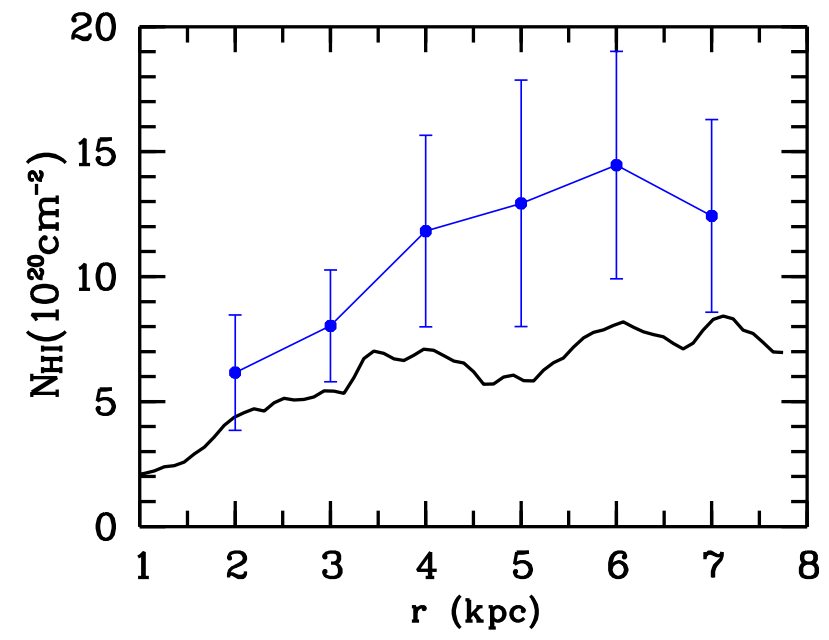

Fig. 6 (online colour at: www.an-journal.org) Radial distributions of $\mathrm{H}$ I column density (thick black curve) and the mean H I column density in H I patches in spiral arms associated with photodissociation regions (blue lines and circles). The $N_{\mathrm{HI}}$ averaged error bars are shown.

the centre (deficiency of $\mathrm{H}$ I is typically observed at the distance of a few kpc from the centre). We examined the radial distribution of $\mathrm{HI}$ column density in NGC 628 based on the $21 \mathrm{~cm}$ line image obtained in Walter et al. (2008) The image 3 was downloaded from the NED data base (Fig. 6). Heiner et al. (2013) estimated atomic hydrogen column densities in H I patches in the spiral arms associated with photodissociation regions in NGC 628. Radial distribution of the mean $\mathrm{H}$ I column density in these H I patches, averaged by $r$, is also presented in Fig. 6 .

As seen from Fig. 6 typical $N_{\mathrm{HI}}$ values in H i patches are 1.5-2 times larger than the values averaged azimuthally. However, their profiles are similar to each other; the $\mathrm{HI}$ column densities increase up to the galactocentric distance $3-4 \mathrm{kpc}$ and they have plateau-like profiles at $r=4-7 \mathrm{kpc}$. These profiles do not coincide with the profiles of the radial distribution of $\langle\mathrm{SFR}\rangle$ and total SFR per galaxy area within star formation regions in spiral arms of the galaxy (Figs. 5 6).

\section{Discussion}

We would like to underline the important result derived in this paper: the peak of the orthogonal component of the velocity of gas inflow into the spiral arms, $v_{\perp}$, coincides with the peak of the radial distribution of the mean SFR within the individual star formation regions. Both peaks are placed within the regular spiral arms at the galactocentric distance $r \approx 4.5-5.0 \mathrm{kpc}$, where the component of the difference between the spiral pattern and gas velocities, that is orthogonal to the arm, is maximal.

\footnotetext{
3 Original image: NGC_628_RO_MOM0:I:HI:wbb2008.fits
}

This result confirms the prediction of the density wave theory illustrated in Roberts et al. (1975). Sakhibov \& Smirnov (2004) showed similar radial distributions of the velocity of gas inflow and the surface density of SFR in another nearby spiral galaxy NGC 6946. The maximum of the velocity of gas inflow and the peak of SFR are at the same radial distance $r \approx 4 \mathrm{kpc}$ from the centre of the galaxy, between the inner Lindblad resonance $(\sim 2.5 \mathrm{kpc})$ and the corotation radius ( $\sim 9 \mathrm{kpc}$ ) (Sakhibov 2004).

Similar situation was noted in the SW arm of M31, where the stellar age gradient across this arm segment is clearly observed, being evident due to the unusually large pitch angle of this arm segment (Efremov 2010), whereas the pitch angle is close to zero for almost all other arms of M31. According to the classical theory (Roberts et al. 1975), the degree of gas compression by a spiral shock wave is determined by the component $v_{\perp}$ of difference between the velocities of solid-body rotation of the density wave $(V d w)$ and differential rotation of the galaxy's gas, $V$, around its centre. The component $v_{\perp}$ is perpendicular to the wave front, i.e. to the inner boundary of the arm: $v_{\perp}=(V-V d w) \sin \mu$ (see Efremov 2010).

It implies that at the same distance from the co-rotation (where $V=V d w$ ), the shock wave should be stronger in the arm segment with the larger pitch angle. The shock wave compresses gas clouds; the higher the density of the initial gas cloud, the higher the effectiveness of star formation. The large pitch angle of the arm segment in question leads to a high value of $v_{\perp}$ (see Fig. 1).

Peaks in the radial distribution of the gas inflow velocity into the spiral arms, as well as in the mean SFR within star formation regions in spiral arms do not coincide with the peak of the radial distribution of $\mathrm{H} \alpha$ luminosity in the galaxy at $r \approx 3 \mathrm{kpc}$. Apparently, this difference may be explained by the radial gradient of dust absorption in the disc of NGC 628 as well as diffuse radiation in $\mathrm{H} \alpha$ in the central part of the galaxy.

On the other hand, the radial distributions of the mean and total SFRs in the individual star formation regions inside and outside the spiral arms significantly differ from each other (Fig. 5]). This result confirms the conclusion made previously by Elmegreen (2011), that the total galactic star formation rate in the main disc is not significantly enhanced by the presence of spiral arms.

The mean surface density of SFR within star formation regions is approximately constant at the distances from 3 to $6 \mathrm{kpc}$ from the centre of NGC 628 (Fig. 4). Obviously, the mean surface density of SFR within star formation regions, $\Sigma_{\text {SFR }}$, depends basically on general properties of interstellar medium such as gas density and pressure.

Both the mean and the total SFRs within star formation regions become equal to each other for the objects inside and outside the spiral arms in the outer part of NGC 628 at $r>5 \mathrm{kpc}$ (Fig.5). These regions are situated in the vicinity of the corotation radius, $R_{\mathrm{cor}} \approx 7 \mathrm{kpc}$, where the velocity of the spiral pattern coincides with the rotation velocity of 
the galaxy. Dynamic properties of interstellar matter inside and outside the spiral arms must be close to each other in this region.

We note in conclusion, that as Martínez-García \& Puerari (2014) have noted, most of a dozen galaxies without signatures of density-formed spiral pattern (Foyle et al. 2011) have spiral arms of the flocculent and multi-arm type (apart from NGC 628 and NGC 5194). These galaxies are not described by the density wave theory.

\section{Conclusions}

We found correlation between the mean SFR within individual star formation regions in the regular parts of spiral arms and the velocity of gas inflow into the spiral arms in the grand-design galaxy NGC 628. Both quantities reach a maximum at a distance of $4.5-5 \mathrm{kpc}$ from the centre of the galaxy.

Radial distributions of SFRs within star formation regions inside and outside the spiral arms are not correlated with each other. However, both the mean and the total SFRs within star formation regions become approximately equal to each other for the objects inside and outside the spiral arms in the outer part of NGC 628 on the distance $r \sim$ $6 \mathrm{kpc}$, near the corotation radius.

The mean surface density of SFR within star formation regions is approximately constant at the distances from 3 to $6 \mathrm{kpc}$ from the centre of NGC 628.

Acknowledgements. We are grateful to the referee for his/her constructive comments. The authors thank A.V. Zasov (SAI MSU) for helpful discussions and E.V. Shimanovskaya (SAI MSU) for help with the editing of this paper. The authors acknowledge the usage of the HyperLeda data base (http://leda.univ-lyon1.fr), the NASA/IPAC Extragalactic Database (http://ned.ipac.caltech.edu), B.A. Miculski archive for space telescopes (http://galex.stsci.edu), and the Padova group online server CMD (http://stev.oapd.inaf.it). This study was supported by the Russian Science Foundation (project no. 14-22-00041).

\section{References}

Aniano, G., Draine, B.T., Calzetti, D., et al. 2012, ApJ, 756, 138

Auld, R., Minchin, R.F., Davies, J.I., et al. 2006, MNRAS, 371, 1617

Belley, J., \& Roy, J.-R. 1992, ApJS, 78, 61

Berg, D.A., Skillman, E.D., Garnett, D.R., Croxall, K.V., Marble, A.R., Smith, J.D., Gordon, K., \& Kennicutt, R.C., Jr. 2013, ApJ, 775, 128

Bresolin, F., Kennicutt, R.C., \& Garnett, D.R. 1999, ApJ, 510, 104

Bruevich, V.V., Gusev, A.S., Ezhkova, O.V., Sakhibov, F.Kh., \& Smirnov, M.A. 2007, Astron. Rep., 51, 222

Calzetti, D., Wu, S.-Y., Hong, S., et al. 2010, ApJ, 714, 1256

Danver, C-G. 1942, Annals of the Observatory of Lund, 10, 3

Disney, M., Davies, J., \& Phillipps S. 1989, MNRAS, 239, 939

Efremov, Yu.N. 2010, MNRAS, 405, 1531

Elmegreen, B.G. 2011, Ecole Evry Schatzman 2010: Star Formation in the Local Universe, Eds. C. Charbonnel \& T. Montmerle, EAS Publications Series, 51, 19
Elmegreen, B.G., \& Elmegreen, D.M. 1983, MNRAS, 203, 31

Fathi, K., Beckman, J.E., Zurita, A., Relaño, M., Knapen, J.H., Daigle, O., Hernandez, O., \& Carignan, C. 2007, A\&A, 466, 905

Ferguson, A.M.N., Gallagher, J.S., \& Wyse, R.F.G. 1998, AJ, 116, 673

Foyle, K., Rix, H.-W., Dobbs, C.L., Leroy, A.K., \& Walter, F. 2011, ApJ, 735, 101

Gusev, A.S., \& Efremov, Yu.N. 2013, MNRAS, 434, 313

Gusev, A.S., Egorov, O.V., \& Sakhibov, F. 2014, MNRAS, 437, 1337

Gusev, A.S., Pilyugin, L.S., Sakhibov, F., Dodonov, S.N., Ezhkova, O.V., \& Khramtsova, M.S. 2012, MNRAS, 424, 1930

Heiner, J.S., Sánchez-Gallego, J.R., Rousseau-Nepton, L., \& Knapen, J.H. 2013, MNRAS, 428, 3355

Hodge, P.W. 1976, ApJ, 205, 728

Iglesias-Páramo, J., Buat, V., Takeuchi, T.T., et al. 2006, ApJS, 164,38

Ivanov, G.R., Popravko, G., Efremov, Yu.N., Tichinov, N.A., \& Karachentsev, I.D. 1992, A\&AS, 96, 645

Kamphuis, J., \& Briggs, F. 1992, A\&A, 253, 335

Kennicutt, R.C. 1998, ARA\&A, 36, 189

Kennicutt, R.C., \& Hodge, P.W. 1976, ApJ, 207, 36

Larsen, S.S. 1999, A\&AS, 139, 393

Larsen, S.S. 2004, A\&A, 416, 537

MacArthur, L.A., González, J.J., \& Courteau, S. 2009, MNRAS, 395, 28

Martínez-García,E.E., \& Puerari,I. 2014, ASPC, 480, 137

McCall, M.L., Rybski, P.M., \& Shields, G.A. 1985, ApJS, 57, 1

Möllenhoff, C. 2004, A\&A, 415, 63

Morgan, W.W., Whitford, A.E., \& Code, A.D. 1953, ApJ, 118, 318

Popescu, C.C., Tuffs, R.J., Dopita, M.A., Fischera, J., Kylafis, N.D., \& Madore, B.F. 2011, A\&A, 527, A109

Roberts, W.W., Roberts, M.S., \& Shu, F.H. 1975, ApJ, 196, 381

Rosales-Ortega, F.F., Diaz, A.I., Kennicutt, R.C., \& Sanchez, S.F. 2011, MNRAS, 415, 2439

Sakhibov F. 2004, D. Sc. Thesis, Lomonosov Moscow State University

Sakhibov, F.Kh., \& Smirnov, M.A. 2004, Astron. Rep., 48, 995

Paturel, G., Petit, C., Prugniel, Ph., Theureau, G., Rousseau, J., Brouty, M., Dubois, P., \& Cambresy, L. 2003, A\&A, 412, 45

Sharina, M.E., Karachentsev, I.D., \& Tikhonov, N.A. 1996, A\&AS, 119, 499

Shostak, G.S., \& van der Kruit, P.C. 1984, A\&A, 132, 20

van Dyk, S.D., Li, W., \& Filippenko, A.V. 2006, PASP, 118, 351

Wyder, T.K., Martin, D.C., Schiminovich, D., et al. 2007, ApJS, 173, 293

Wakker, B.P., \& van Woerden, H. 1991, A\&A, 250, 509

Walter, F., Brinks, E., de Blok, W.J.G., Bigiel, F., Kennicutt, Jr. R.C., Thornley, M.D., \& Leroy, A. 2008, AJ, 136, 2563 